\title{
Compressed Sensing for THz FMCW Radar 3D Imaging
}

\author{
Shanshan Gu $\mathbb{D},{ }^{1}$ Guangrong $X i \mathbb{D}^{2},{ }^{2}$ Lingyu Ge $\mathbb{D}^{\mathbb{D}},{ }^{3}$ Zhong Yang $\mathbb{D},{ }^{1}$ Yizhi Wang $\mathbb{D},{ }^{1}$ \\ Weina Chen ${ }^{1}{ }^{1}$ and Zhenzhong Yu ${ }^{1}$ \\ ${ }^{1}$ College of Intelligent Science and Control Engineering, Jinling Institute of Technology, Nanjing 211169, China \\ ${ }^{2}$ Shanghai Geometrical Perception and Learning Co., Ltd., Shanghai 201206, China \\ ${ }^{3}$ Nanjing Metro Group Co., Ltd., Nanjing 210018, China
}

Correspondence should be addressed to Zhong Yang; yz@jit.edu.cn

Received 1 March 2021; Accepted 17 August 2021; Published 27 August 2021

Academic Editor: Jing $\mathrm{Na}$

Copyright (C) 2021 Shanshan Gu et al. This is an open access article distributed under the Creative Commons Attribution License, which permits unrestricted use, distribution, and reproduction in any medium, provided the original work is properly cited.

A terahertz $(\mathrm{THz})$ frequency-modulated continuous wave (FMCW) imaging radar system is developed for high-resolution 3D imaging recently. Aiming at the problems of long data acquisition periods and large sample sizes for the developed imaging system, an algorithm based on compressed sensing is proposed for THz FMCW radar 3D imaging in this paper. Firstly, the FMCW radar signal model is built, and the conventional range migration algorithm is introduced for THz FMCW radar imaging. Then, compressed sensing is extended for THz FMCW radar 3D imaging, and the Newton smooth L0-norm (NSL0) algorithm is presented for sparse measurement data reconstruction. Both simulation and measurement experiments demonstrate the feasibility of reconstructing $\mathrm{THz}$ images from measurements even at the sparsity rate of $20 \%$.

\section{Introduction}

Terahertz $(\mathrm{THz})$ wave lies between infrared wave and millimeter wave, which is an electromagnetic wave that has not been fully recognized and utilized by human beings. Due to its ability of material penetration and harmless nonionizing radiation to human body, $\mathrm{THz}$ technology can be employed to effectively identify stealth and deceptive measures that cannot be distinguished by conventional means in the military fields and to identify concealed objects made of metal or inorganic materials for security check. As THz has a smaller wavelength and a wider bandwidth which will result in a higher resolution, $\mathrm{THz}$ imaging has been widely used in nondestructive inspections and medical diagnosis $[1,2]$. With the rapid development of information countermeasures, antistealth, target search, and tracking, and materials science, $\mathrm{THz}$ imaging technology has made a great progress during the past decades.

Motivated by the huge application potential of highresolution $\mathrm{THz}$ imaging technology, there has been growing interest in developing 3D imaging radar working at $\mathrm{THz}$ range. In 2007, a $220 \mathrm{GHz}$ experimental frequency- modulated continuous wave (FMCW) inverse synthetic aperture radar (SAR) with a bandwidth of $8 \mathrm{GHz}$ is designed to determine high-resolution scattering center distributions of targets [3]. A $240 \mathrm{GHz} 3 \mathrm{D}$ FMCW imaging radar with a maximum bandwidth of $42 \mathrm{GHz}$ is discussed in [4]. An imaging radar with the operation frequency of $580 \mathrm{GHz}$ which is implemented in an all-solid-state design is developed at Jet Propulsion Laboratory (JPL) [5]. The first THz radar for fast standoff personnel screening with the operation frequency of $675 \mathrm{GHz}$ is also built by JPL. A fast scanning device is designed to enable imaging at a frame rate of $1 \mathrm{~Hz}[6,7]$. An active FMCW imaging system ranging from 514 to $565 \mathrm{GHz}$ (frequency centered at $540 \mathrm{GHz}$ ) is studied to image objects with a resolution of millimeter [8].

It can be found that $\mathrm{THz} 3 \mathrm{D}$ imaging is commonly realized with SAR technique, rather than building antenna arrays. This is due to that expensive devices in $\mathrm{THz}$ regime will lead to a high-cost imaging system having multiple transceivers. For a THz imaging system with SAR technique, the single transceiver is moved in a grid-like manner to produce an image with the processing approaches. Several SAR imaging algorithms in time domain, frequency domain, 
and wavenumber domain have been proposed for $\mathrm{THz}$ FMCW SAR, respectively. A typical time domain backprojection algorithm is studied to obtain the image with $2 \mathrm{D}$ aperture synthesis for the SynView $\mathrm{THz} 3 \mathrm{D}$ imaging system [9]. A back-projection imaging approach has been presented in [10] for data processing of a $300 \mathrm{GHz}$ imaging system. However, imaging algorithms in the time domain have a heavy calculation burden though they are able to process SAR data under a great variety of imaging geometries. A revised range-Doppler algorithm is presented for FMCW SAR imaging by compensating radar migration [11]. A nonlinear frequency-scaling algorithm is proposed by Meta et al. to achieve FMCW SAR focusing by applying Dopplerrange correction [12]. However, the range-Doppler algorithm and the nonlinear frequency-scaling algorithm in frequency domain will result in deviations due to the highorder phase error. So, the range migration algorithm (RMA) in wavenumber domain is preferable for $\mathrm{THz}$ FMCW SAR imaging $[8,13]$.

As mentioned above, a single transceiver is moved in a grid-like manner to acquire data for $\mathrm{THz}$ FMCW SAR imaging in most cases. And the mechanical scanning parameters should obey the Nyquist-Shannon sampling theorem for the RMA imaging algorithm. However, a smaller wavelength in $\mathrm{THz}$ regime requires a smaller scanning step, and it will lead to a larger collection points and a longer data acquirement time. The $\mathrm{THz}$ imaging efficiency is low for the conventional imaging algorithms. To reduce imaging data acquirement time and to improve imaging efficiency, an algorithm based on compressed sensing is proposed for 3D imaging of $\mathrm{THz}$ FMCW SAR in this paper. The proposed algorithm relies on the advantages of compressed sensing that it can reconstruct signal from the sparse data samples.

The paper is organized as follows. The developed $220 \mathrm{GHz}$ FMCW SAR imaging system is introduced in Section 2. And the RMA for THz FMCW radar imaging is given in Section 3. Section 4 describes the proposed 3D imaging algorithm based on compressed sensing. The experimental results are given and analyzed in Section 5 to verify the proposed imaging algorithm. The last section is Conclusion.

\section{THz FMCW Imaging System}

2.1. System Briefs. As shown in Figure 1, the FMCW THz imaging system developed in this paper consists of radio frequency transceiver subsystem, signal acquisition and processing subsystem, and planar scanning subsystem.

When the THz FMCW imaging system works, an $\mathrm{X}$-band FMCW signal generated through the direct digital synthesizer serves as the driving source of the transmission link which will output a THz FMCW signal. Then, the transmitted signal will be reflected by target and then received by the antenna. The received echoed signal is mixed with the reference signal to get the intermediate frequency (IF) signal which will be processed with the IF processing unit. Lastly, the processed IF signal is acquired with the data acquisition unit. The planar scanner controlled by the data acquisition and processing subsystem moves with a "stopgo-stop" manner. The echoed signals at certain points are acquired and stored by the processing software and are processed to generate 3D images after finalization of scanning. The picture of the developed THz FMCW imaging system is presented in Figure 2. And the specification parameters are illustrated in Table 1.

2.2. THz FMCW Signal Modeling. The transmitted signal of the THz FMCW imaging system can be expressed as

$$
s_{t}(t)=\exp \left(j \pi k_{r} t^{2}\right) \exp \left(j 2 \pi f_{c} t\right),
$$

where $f_{c}$ denotes the central frequency, $T$ means the sweep duration, and $k_{r}$ is the chirp frequency rate. Assumed that the distance between the target and the radar is $R$, the echoed signal can be expressed as

$$
s_{r}\left(t-\tau_{R}\right)=g \cdot \exp \left[j \pi k_{r}\left(t-\tau_{R}\right)^{2}\right] \exp \left[j 2 \pi f_{c}\left(t-\tau_{R}\right)\right],
$$

where $\tau_{R}=2 R / c$ is the echoed signal delay and $g$ represents the target reflection coefficient. Dechirp signal processing technique is adopted in the developed imaging system to obtain IF signal. That is, IF signal is output by mixing the transmitted signal and the echoed signal, and it can be written as

$$
s(t)=g \cdot \exp \left[-j \pi k_{r}\left(2 \tau_{R} t-\tau_{R}^{2}\right)\right] \exp \left(-j 2 \pi f_{c} \tau_{R}\right),
$$

where $\pi k_{r} \tau_{R}^{2}$ is the residual phase error (RVP) after dechirp. In general, this item can be ignored for imaging. Then, equation (3) can be simplified as

$$
\begin{aligned}
s(t) & =g \cdot \exp \left[-j 2 \pi\left(k_{r} t+f_{c}\right) \tau_{R}\right], \\
& =g \cdot \exp \left(-j 2 \pi f \tau_{R}\right),
\end{aligned}
$$

where $f=k_{r} t+f_{c}$, so the above expression can be rewritten as

$$
s(t)=g \cdot \exp \left(-\frac{j 4 \pi f R}{c}\right)
$$

\section{Range Migration Algorithm for THz FMCW Radar Imaging}

Though several algorithms have been developed for $\mathrm{THz}$ FMCW radar imaging, RMA in the wave number domain is widely used due to a higher efficiency. The imaging geometry and RMA for THz FMCW radar will be introduced in this section.

The imaging geometry of the developed imaging system is presented in Figure 3. The transceiver mounted on the planar scanner is controlled to move with a grid-like manner, which will lead to a $2 \mathrm{D}$ rectangular synthetic aperture formation $e$ on the $X^{\prime} O^{\prime} Y^{\prime}$ plane which is parallel to the XOY plane.

For the convenience of expression, equation (5) is rewritten as 


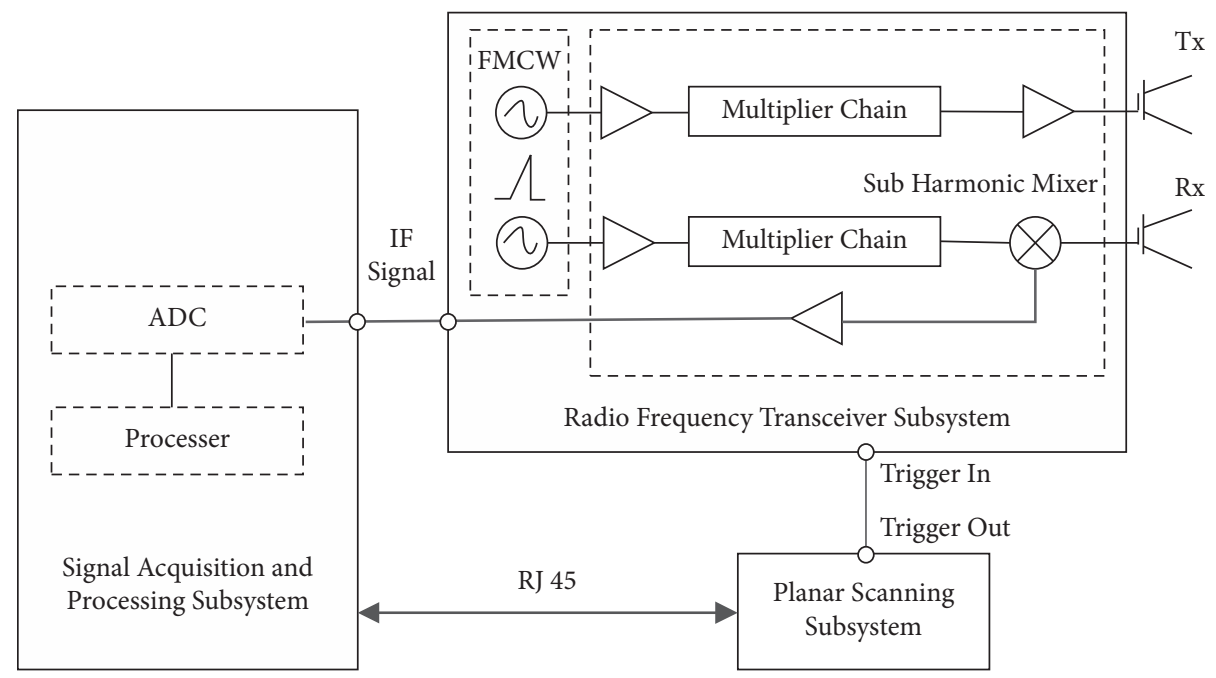

FIgURE 1: Schematic diagram of the developed THz FMCW imaging system.

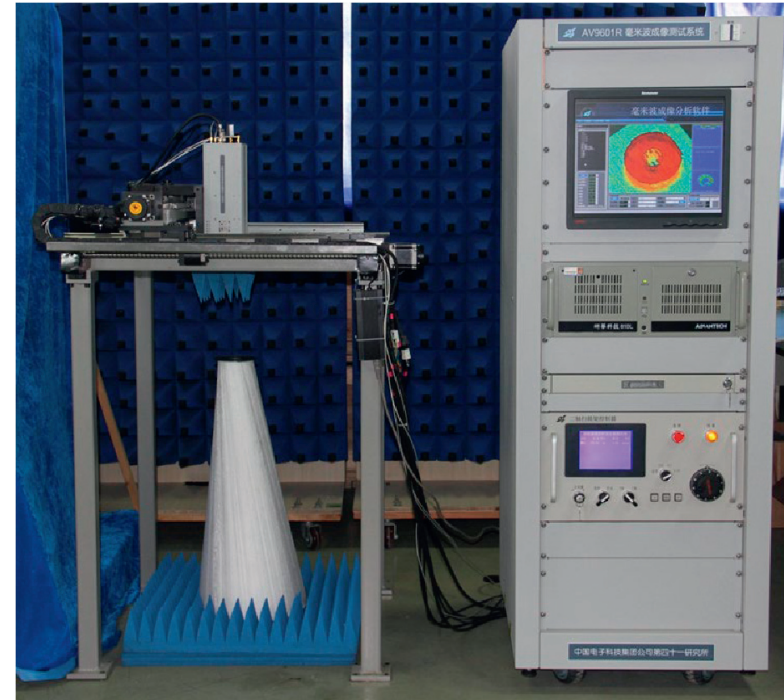

Figure 2: Picture of the developed THz FMCW imaging system.

$$
s(t)=g \cdot e^{-j 2 k R},
$$

where $k=2 \pi f / c$ and $R=\sqrt{\left(x^{\prime}-x\right)^{2}+\left(y^{\prime}-y\right)^{2}+\left(z_{0}-z\right)^{2}}$ is the range between the measurement point $\left(x^{\prime}, y^{\prime}, z_{0}\right)$ and the scattering center $(x, y, z)$. Then, the sum of all received echoed signals at a measurement point $\left(x^{\prime}, y^{\prime}, z_{0}\right)$ within the imaging area is

$$
s\left(x^{\prime}, y^{\prime}, k\right)=\iiint_{P} g(x, y, z) e^{-j 2 k R} \mathrm{~d} x \mathrm{~d} y \mathrm{~d} z,
$$

where $P$ denotes the imaging area and $g(x, y, z)$ represents the reflection coefficient matrix of target.

Applying two-dimensional Fourier transform of the received echoed signals along the scanning direction,
TABLE 1: The developed THz FMCW imaging system parameters.

\begin{tabular}{lc}
\hline Parameters & Value \\
\hline Center frequency & $195 \mathrm{GHz}$ \\
Bandwidth & $50 \mathrm{GHz}$ \\
Output power & $>-2 \mathrm{dBm}$ \\
Receiver sensitivity & $-100 \mathrm{dBm}$ \\
Sweep duration & $0.1-1 \mathrm{~ms}$ \\
Maxim scanning area & $500 \mathrm{~mm}^{*} 500 \mathrm{~mm}$ \\
\hline
\end{tabular}

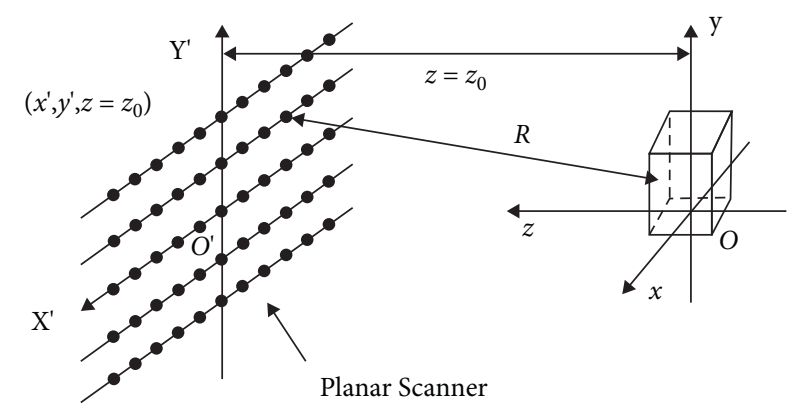

FIGURE 3: Imaging geometry of the developed THz FMCW imaging radar system.

$$
\begin{aligned}
S\left(k_{x}, k_{y}, k\right)= & \iiint \iint_{P} g(x, y, z) e^{-j 2 k R} \mathrm{~d} x \mathrm{~d} y \mathrm{~d} z \\
& \cdot e^{-j k_{x} x^{\prime}} e^{-j k_{y} y^{\prime}} \mathrm{d} x^{\prime} \mathrm{d} y^{\prime}, \\
= & \iiint_{P} g(x, y, z) S_{0}\left(k_{x}, k_{y}, k\right) \mathrm{d} x \mathrm{~d} y \mathrm{~d} z
\end{aligned}
$$

where $k_{x}$ and $k_{y}$ represent the spatial frequencies in the $X$ and $Y$-directions and $S_{0}\left(k_{x}, k_{y}, k\right)=\iint e^{-j 2 k R} e^{-j k_{x} x^{\prime}}$ $e^{-j k_{y} y^{\prime}} \mathrm{d} x^{\prime} \mathrm{d} y^{\prime}$. And $S_{0}\left(k_{x}, k_{y}, k\right)$ can be solved as 


$$
S_{0}\left(k_{x}, k_{y}, k\right)=e^{j \sqrt{4 k^{2}-k_{x}^{2}-k_{y}^{2}}\left(z_{0}-z\right)} e^{-j k_{x} x} e^{-j k_{y} y} .
$$

Considering that $k_{x}^{2}+k_{y}^{2}+k_{z}^{2}=4 k^{2}$, equation (8) can be rewritten as

$$
S\left(k_{x}, k_{y}, k\right)=\iiint_{P} g(x, y, z) e^{j \sqrt{4 k^{2}-k_{x}^{2}-k_{y}^{2}} z_{0}} e^{-j k_{x} x} e^{-j k_{y} y} e^{-j k_{z} z} \mathrm{~d} x \mathrm{~d} y \mathrm{~d} z .
$$

After multiplying the reference function $e^{-j \sqrt{4 k^{2}-k_{x}^{2}-k_{y}^{2}} z_{0}}$, we can obtain the following expression:

$$
S_{1}\left(k_{x}, k_{y}, k\right)=\iiint_{P} g(x, y, z) e^{-j k_{x} x} e^{-j k_{y} y} e^{-j k_{z} z} \mathrm{~d} x \mathrm{~d} y \mathrm{~d} z .
$$

It can be found from equation (11) that $S_{1}\left(k_{x}, k_{y}, k\right)$ is the Fourier transform of $g(x, y, z)$, so the reflection coefficient matrix $g(x, y, z)$ which corresponds to the image of target can be derived by applying inverse Fourier transform. However, the measured data are not uniformly distributed in the $k_{z}$ domain due to a nonlinear conversion from $k$ to $k_{z}$. Generally, the Stolt interpolation method is used to obtain a uniformly distributed data in the $k_{z}$ domain. Finally, the reflection coefficient matrix $g(x, y, z)$ can be obtained with

$$
\begin{aligned}
g(x, y, z)= & \operatorname{IFFT}_{\left(k_{x}, k_{y}, k_{z}\right)} \\
& \cdot\left\{\operatorname{stolt}_{k \longrightarrow k_{z}}\left[S\left(k_{x}, k_{y}, k\right) e^{j \sqrt{4 k^{2}-k_{x}^{2}-k_{y}^{2}} z_{0}}\right]\right\} .
\end{aligned}
$$

The THz FMCW SAR imaging algorithm based on RMA can be summarized as follows:

(1) 2D Fourier transform is applied on the data collected by planar scanning to obtain wavenumber domain formulation $S\left(k_{x}, k_{y}, k\right)$

(2) The reference function $e^{-j \sqrt{4 k^{2}-k_{x}^{2}-k_{y}^{2}} z_{0}}$ is multiplied at the reference range $z_{0}$

(3) Stolt interpolation is performed to generate data which are uniformly distributed in the $k_{z}$ domain

(4) Finally, 3D inverse Fourier transform is performed to produce the image of target.

\section{Imaging Algorithm Based on Compressed Sensing}

4.1. Compressed Sensing Principle. Compressed sensing is a signal processing technique which is able to realize the recovery of a sparse signal with fewer samplers required by the Nyquist sampling theorem. In view of the advantages of compressed sensing, this technique is investigated for $\mathrm{THz}$ FMCW SAR imaging to reduce the requirement for data sampling and to increase the imaging speed.

Suppose $g$ is a discrete signal with a length of $N$ in the time domain, and it can be represented linearly by a set of orthonormal basis as

$$
\boldsymbol{g}=\boldsymbol{\Psi} x=\sum_{i=1}^{N} \boldsymbol{\Psi}_{i} x_{i}
$$

where $\Psi=\left\{\Psi_{1}, \ldots, \Psi_{N}\right\}$ is the sparse transformation basis and $x=\left\{x_{1}, \ldots, x_{N}\right\}$ is the weighting coefficients of $g$ satisfying that $x_{i}=\Psi_{i}^{T} g$.

It can be seen from equation (13) that $x$ is the equivalent representation of $g$. If there are only $K$ nonzero elements in $x$, then $x$ is the $K$-sparse representation of signal $g$, and the signal sparsity is $K$.

Generally, the received $\mathrm{THz}$ FMCW radar signal is nonsparse in the time domain. So, it is necessary to transform the nonsparse time domain signal to the sparse transform domain. Fourier transform is employed here for signal transform operations in this paper. Compressed sampling is realized with a measurement matrix $\phi$ which projects high-dimensional signal on the low-dimensional space:

$$
y=\phi g=\phi \Psi x=A x,
$$

where $y$ is the vector of measurements of the original highdimensional signal $g$ under a random matrix $\phi, \Psi$ is the sparse basis matrix, and $A=\phi \Psi$ is the sensing matrix with a dimension of $M \times N(M \ll N)$.

The $N$-dimensional signal $x$ can be recovered from the $M$-dimensional measurement data through signal reconstruction. And the signal reconstruction is realized by solving the $L_{0}$-norm minimization problem expressed as

$$
\begin{aligned}
\widehat{x} & =\arg \min \|x\|_{0}, \\
\text { s.t. } y & =A x .
\end{aligned}
$$

Though the above minimum $L_{0}$-norm is an NP-hard problem which cannot be solved directly, an optimal solution can be realized with greedy search or convex optimization algorithms.

4.2. THz FMCW Radar Imaging Algorithm Based on Compressed Sensing. Because the recovery accuracy of greedy search reconstruction algorithms like orthogonal matching pursuit (OMP) $[14,15]$, stage-wise orthogonal matching pursuit (StOMP) [16], regularized orthogonal matching pursuit (ROMP) [17], and compressive sampling matching pursuit (CoSaMP) [18] is poor with lower signal noise ratio (SNR), an improved smoothed $L_{0}$-norm minimization (SL0) algorithm based on the convex optimization is presented in this paper.

For the SL0 algorithm, the objective function is defined as follows [19, 20]:

$$
\begin{aligned}
& \arg \min F_{\sigma}(x), \\
& \text { s.t. } y=A x, \\
& \|x\|_{0}=\lim _{\sigma \longrightarrow 0} F_{\sigma}(x)=\lim _{\sigma \longrightarrow 0} \sum_{i=1}^{N} F_{\sigma}\left(x_{i}\right),
\end{aligned}
$$

where $F_{\sigma}(x)$ is a smoothed function, which can be regarded as $\|x\|_{0}$ when $\sigma$ is close to 0 . It is obvious that the smoothed function $F_{\sigma}(x)$ will approximate to the optimal solution by choosing a suitable $\sigma$. And $F_{\sigma}\left(x_{i}\right)$ is defined as 


$$
F_{\sigma}\left(x_{i}\right)=\frac{\exp \left(x_{i}^{2} / 2 \sigma^{2}\right)-\exp \left(-x_{i}^{2} / 2 \sigma^{2}\right)}{\exp \left(x_{i}^{2} / 2 \sigma^{2}\right)+\exp \left(-x_{i}^{2} / 2 \sigma^{2}\right)} .
$$

Compared with the Gauss smoothed function, the presented smoothed function will get a better performance in signal reconstruction as this leads to a closer approximation to $\|x\|_{0}$.

The steepest descent algorithm is commonly applied to solve equation (16). However, it is difficult to estimate the optimal searching step in the algorithm, and this will lead to a slower convergence speed. A revised Newton method in [21] is utilized in this paper to solve the optimization problem more efficiently. The Newton directions is revised as

$$
d=-G^{-1} \nabla F_{\sigma}(x),
$$

where $\quad \nabla F_{\sigma}(x)=\left[\left(\partial f_{\sigma}\left(x_{1}\right) / \partial x_{1}\right), \ldots,\left(\partial f_{\sigma}\left(x_{N}\right) / \partial x_{N}\right)\right]^{T}$, $G=\nabla^{2}\left(F_{\sigma}(x)\right)+\varepsilon_{k} \mathbf{I}, \mathbf{I}$ is identity matrix, and $\varepsilon_{k}$ is positive to make sure that diagonal values of $G$ are also positive. Here, $\varepsilon_{k}$ is set as

$$
\varepsilon_{k}=\frac{4 / \sigma^{2}\left[\left(3 x_{i}^{2} / \sigma^{2}\right) \exp \left(-x_{i}^{2} / 2 \sigma^{2}\right)-\left(x_{i}^{2} / \sigma^{2}\right) \exp \left(x_{i}^{2} / 2 \sigma^{2}\right)\right]}{\left[\exp \left(-x_{i}^{2} / 2 \sigma^{2}\right)+\exp \left(-x_{i}^{2} / 2 \sigma^{2}\right)\right]^{3}} \text {. }
$$

So, the revised Newton directions can be written as

$$
d=\left(-\frac{\sigma^{2} x_{1}}{\sigma^{2}+x_{1}^{2}}, \ldots,-\frac{\sigma^{2} x_{i}}{\sigma^{2}+x_{i}^{2}}, \ldots,-\frac{\sigma^{2} x_{n}}{\sigma^{2}+x_{n}^{2}}\right)^{T} .
$$

The realization steps of the presented sparse signal reconstruction algorithm based on the Newton smooth $L_{0}$-norm (NSLO) are summarized in Table 2.

Initialization:

(1) $x_{0}=A^{T}\left(A A^{T}\right)^{-1} y$.

(2) Choose a suitable decreasing sequence for $\sigma$, $\left\{\sigma_{1}, \sigma_{2}, \ldots, \sigma_{j}\right\}, \sigma_{j}=\beta \sigma_{j-1}, \beta(0<\beta<1)$ is the decreasing factor.

Realization:

(1) For $j=1,2,3, \ldots, J$

$$
\begin{aligned}
& \text { A, } \sigma=\sigma_{j} ; \\
& \text { B, } x_{j}=x_{j-1} ; \\
& \text { C, } r_{0}=0 ; \\
& D, \text { For } n=1,2,3, \ldots, N
\end{aligned}
$$

(a) Calculate the Newton direction $d=\left(-\left(\sigma^{2} x_{1} / \sigma^{2}+x_{1}^{2}\right), \ldots,-\left(\sigma^{2} x_{n} / \sigma^{2}+x_{n}^{2}\right)\right)$

(b) Update $x_{j_{n}} \leftarrow x_{j_{n}}+d$

(c) Project $x$ back onto the feasible set

$$
x_{j_{n}} \leftarrow x_{j_{n}}-A^{T}\left(A A^{T}\right)^{-1}\left(A x_{j_{n}}-y\right)
$$

(d) Let $r=y-A x_{j_{n}}$;

(e) If $\left\|r-r_{0}\right\|<e$, break; Else, $r_{0}=r$;

$$
E, x_{j}=x_{j_{n}} \text {; }
$$

(2) Final answer is $\widehat{x}=x_{j}$.
TABLE 2: NSL0 algorithm.

\begin{tabular}{lcc}
\hline & Algorithm & \\
Sparsity rate (\%) & SL0 & NSL0 \\
\hline 10 & 7.8157 & 6.8796 \\
20 & 11.6065 & 10.6881 \\
30 & 17.2381 & 16.3765 \\
40 & 24.3518 & 23.1646 \\
50 & 32.8382 & 31.9270 \\
\hline
\end{tabular}

Based on the above sparse signal reconstruction algorithm, the 3D imaging algorithm of THz FMCW SAR based on compressed sensing proposed in this paper can be summarized as follows:

(1) Design a measurement matrix $\phi$ which is able to meet the requirement for data acquisition

(2) Collect THz FMCW SAR echoed signal at the corresponding position given by the designed measurement matrix to obtain measurement signal $y$

(3) Reconstruct original signal $\hat{g}$ from the sparse measurement data using the presented NSL0 algorithm

(4) Apply 2D Fourier transform with respect to the reconstructed signal $\hat{g}$

(5) Perform reference function multiplying (RFM)

(6) Perform Stolt interpolation

(7) Perform 3D inverse Fourier transform to generate the image

The proposed $\mathrm{THz}$ FMCW SAR imaging algorithm based on compressed sensing is shown in Figure 4.

\section{Experimental Result}

Simulation and measurement experiments are performed to verify the presented THz FMCW SAR imaging algorithm in this paper.

5.1. Point Targets Simulation. The THz FMCW SAR imaging model is built with MATLAB, and six-point targets are simulated for imaging. The coordinates of targets are $(0.2 \mathrm{~m}, 0 \mathrm{~m}, 0 \mathrm{~m}), \quad(-0.2 \mathrm{~m}, 0 \mathrm{~m}, 0 \mathrm{~m}), \quad(0 \mathrm{~m}, 0.2 \mathrm{~m}, 0 \mathrm{~m})$, $(0 \mathrm{~m},-0.2 \mathrm{~m}, 0 \mathrm{~m}), \quad(0 \mathrm{~m}, 0 \mathrm{~m}, 0.2 \mathrm{~m})$, and $(0 \mathrm{~m}, 0 \mathrm{~m},-0.2 \mathrm{~m})$. The imaging radar system parameters used in the simulation are listed in Table 3. The measurement matrix used in the simulation is a sparse random matrix, and the sparse sampling rate is $50 \%$.

The sparse simulated data are firstly recovered with compressed sensing. Then, the recovered signal is processed with RMA to produce a $3 \mathrm{D}$ image as shown in Figure 5(a). Also, the imaging results with the full data using RMA are presented in Figure 5(b) for comparison.

It can be seen from the figures that the produced 3D images are almost the same. And, it shows that the presented THz FMCW SAR imaging algorithm works well with the sparse measurement. 


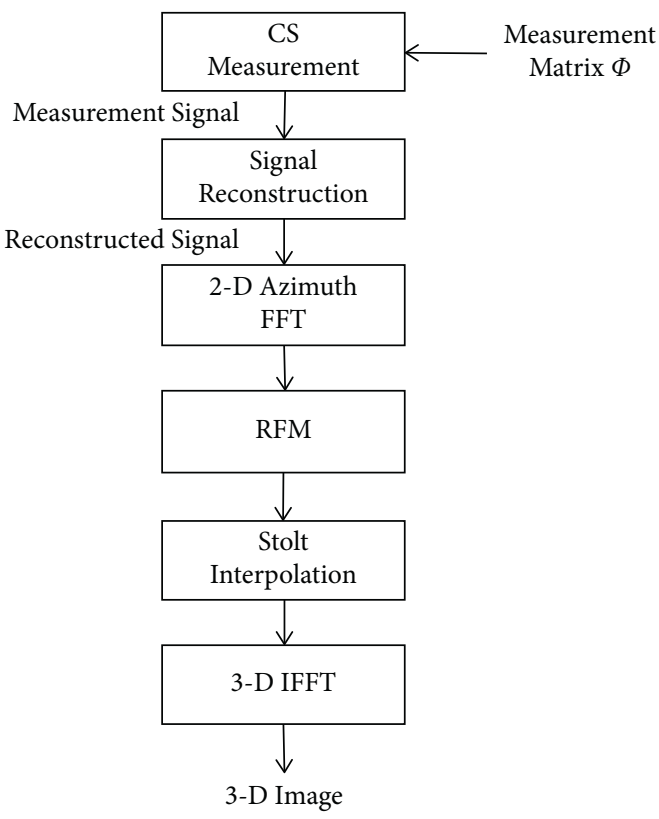

Figure 4: THz FMCW SAR imaging algorithm based on compressed sensing.

TABLE 3: Simulation parameters.

Frequency range

$170 \mathrm{GHz}-220 \mathrm{GHz}$

Bandwidth

$50 \mathrm{GHz}$

Distance $z_{0}$

$0.5 \mathrm{~m}$

Scanning step along $X I$-axis

$0.001 \mathrm{~m}$

Scanning step along $Y /$-axis

$0.001 \mathrm{~m}$

Scanning points along $X /$-axis

1000

Scanning points along $Y /$-axis

1000

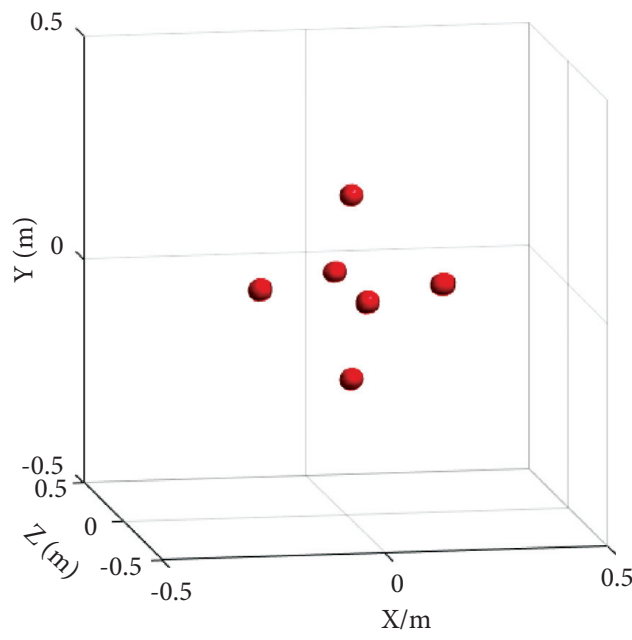

(a)

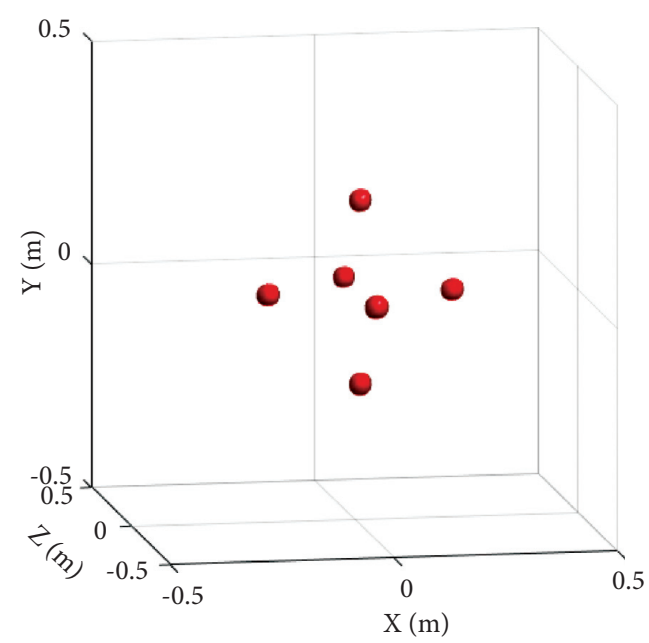

(b)

FIGURE 5: Reconstructed 3D images of point targets: (a) presented algorithm in this paper; (b) RMA. 


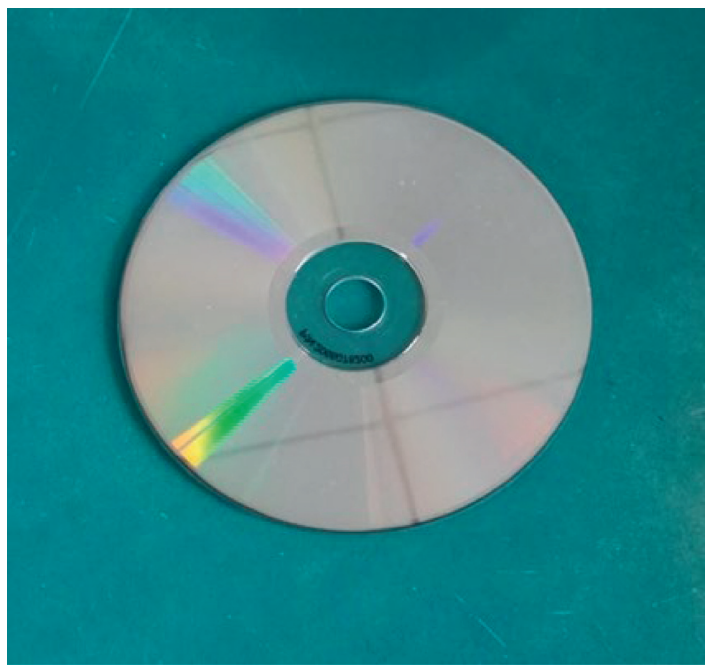

(a)

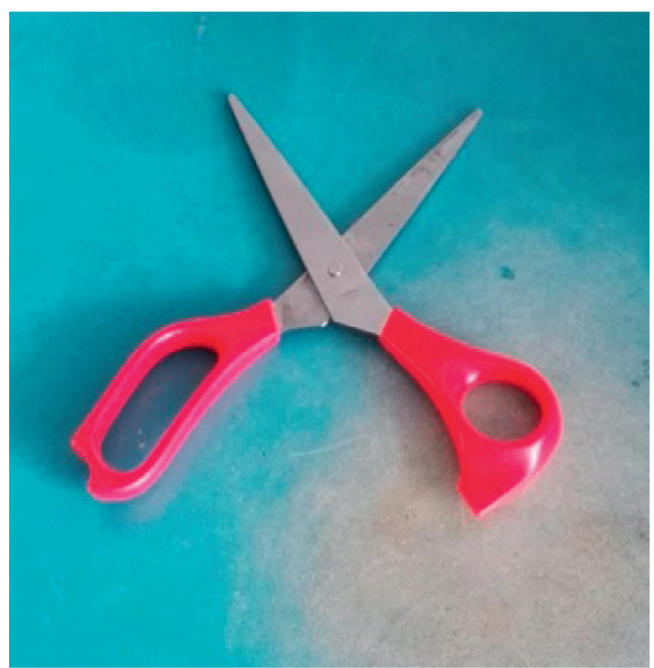

(b)

Figure 6: Targets for imaging experiments: (a) disc; (b) scissors.

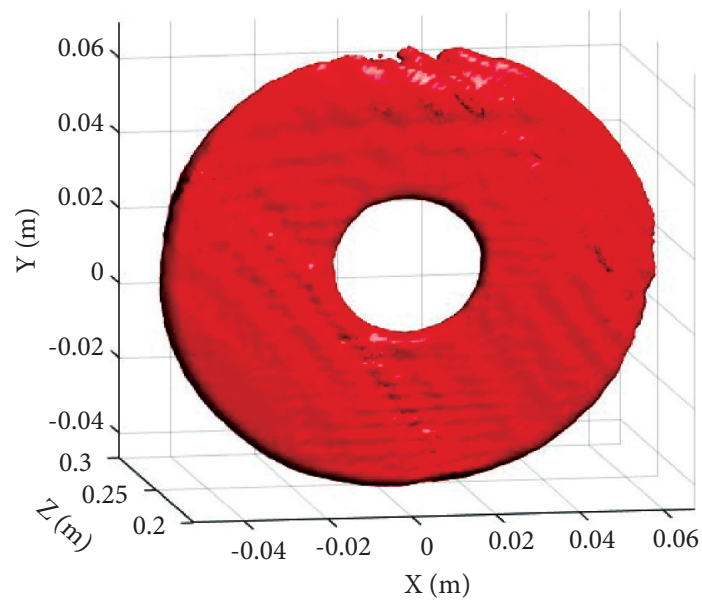

(a)

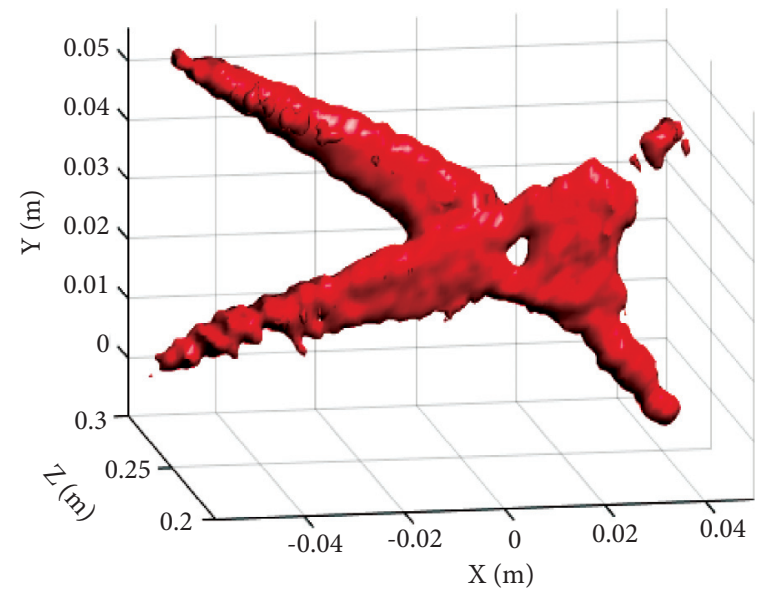

(b)

Figure 7: Reconstructed 3D images of targets with RMA: (a) disc; (b) scissors.

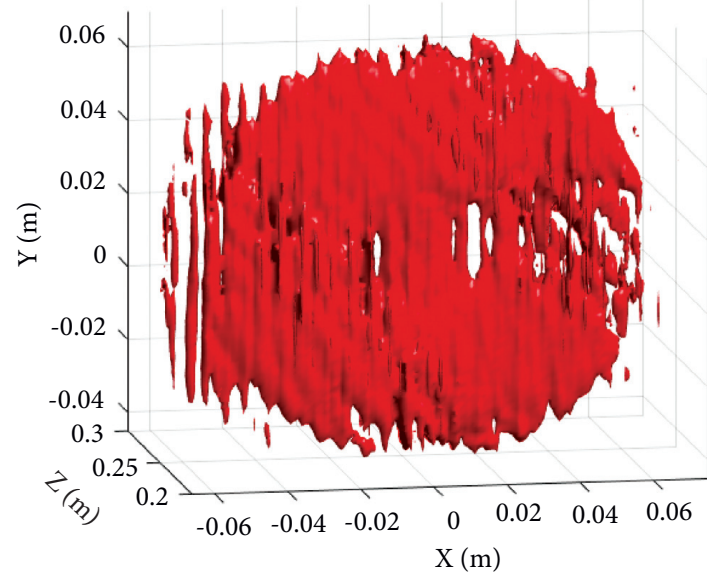

(a)

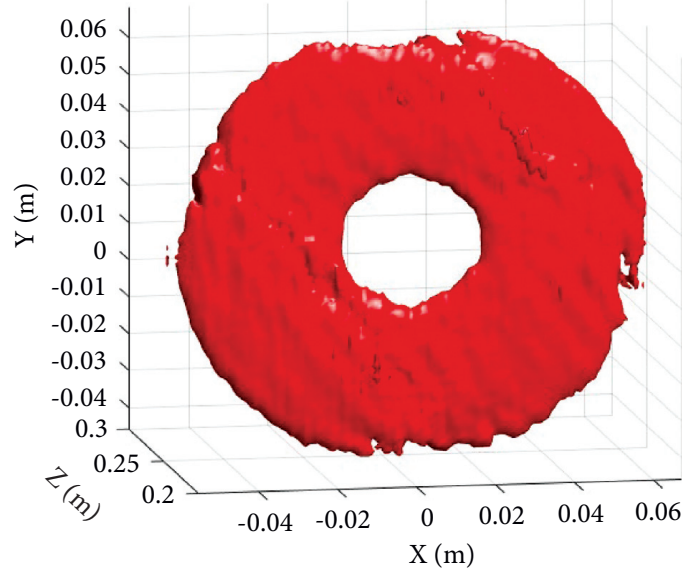

(b)

Figure 8: Continued. 


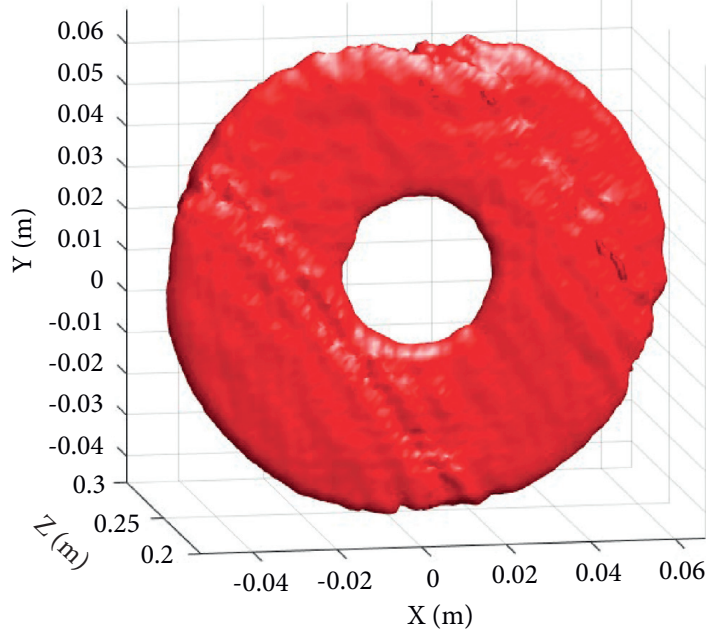

(c)

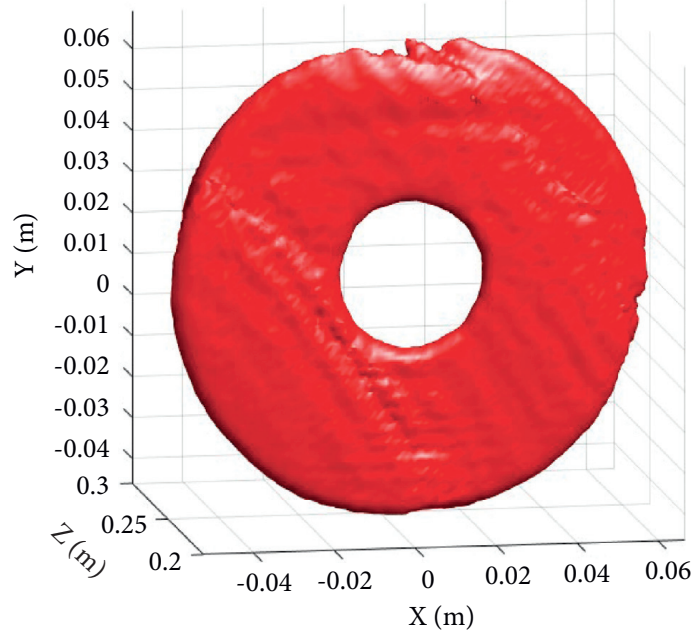

(d)

FiguRE 8: Reconstructed 3D images of disc with the presented algorithm under different data sparsity rates: (a) $10 \%$; (b) $20 \%$; (c) $30 \%$; (d) $50 \%$.

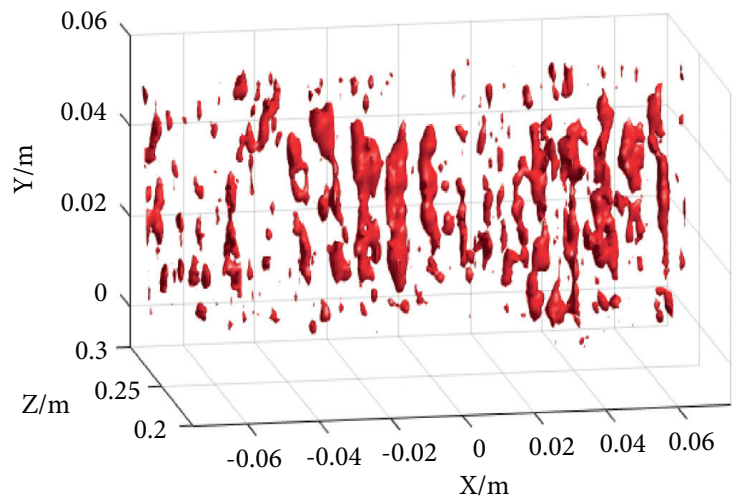

(a)

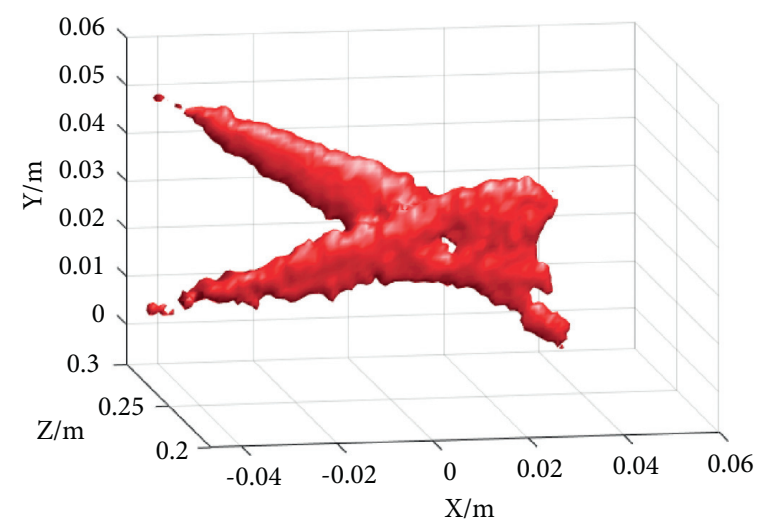

(c)

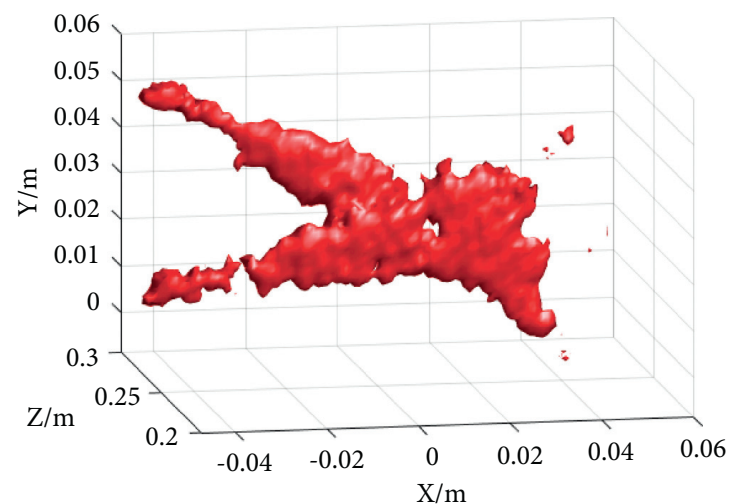

(b)

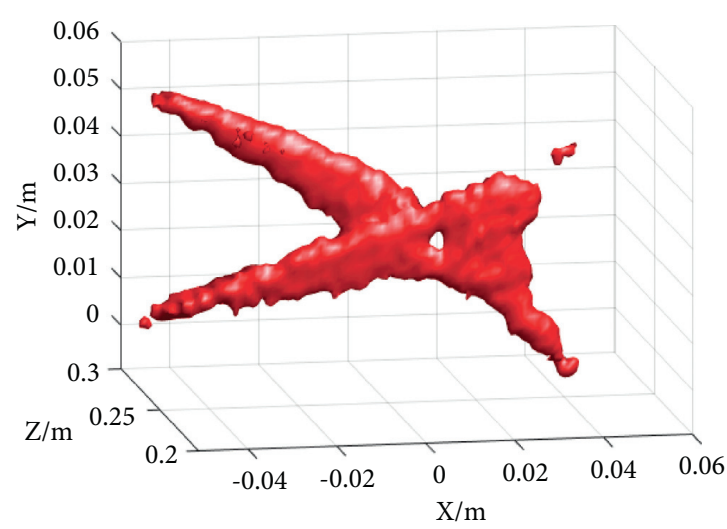

(d)

FiguRE 9: Reconstructed 3D images of scissors with the presented algorithm under different data sparsity rates: (a) 10\%; (b) 20\%; (c) 30\%; (d) $50 \%$.

5.2. Measurement Experiment. In order to further verify the proposed 3D imaging algorithm for THz FMCW SAR, experiments are conducted with the developed $\mathrm{THz}$ FMCW imaging radar system. The operation frequency range of the system is $170-220 \mathrm{GHz}$. The targets to be imaged are placed on a foam column which is $0.5 \mathrm{~m}$ away from the planar 
TABLE 4: Reconstruction error comparisons between SL0 and NSLO.

\begin{tabular}{lcc}
\hline & Algorithm & \\
Sparsity rate (\%) & SL0 & NSL0 \\
\hline 10 & 0.8627 & 0.8354 \\
20 & 0.6447 & 0.6265 \\
30 & 0.4835 & 0.4693 \\
40 & 0.3545 & 0.3423 \\
50 & 0.2757 & 0.2641 \\
\hline
\end{tabular}

TABLE 5: Calculation time comparisons between SL0 and NSLO $(s)$.

\begin{tabular}{lcc}
\hline & Algorithm & \\
Sparsity rate (\%) & SL0 & NSL0 \\
\hline 10 & 7.8157 & 6.8796 \\
20 & 11.6065 & 10.6881 \\
30 & 17.2381 & 16.3765 \\
40 & 24.3518 & 23.1646 \\
50 & 32.8382 & 31.9270 \\
\hline
\end{tabular}

scanning plane, and the scanning area is $151 \mathrm{~mm} \times 151 \mathrm{~mm}$. The planar scanner moves with a "stop-go-stop" manner with a scanning step of $1 \mathrm{~mm}$. The collected full data are processed to reconstruct the image of target with RMA first. The reconstructed images of a disc and a pair of scissors as shown in Figure 6 are presented in Figure 7. Then, 10\%, 20\%, $30 \%$, and $50 \%$ of the collected data are extracted according to the measurement matrix $\phi$. The extracted sparse data are then processed with the proposed compressed sensing imaging algorithm. The reconstructed images under different sparsity rates are shown in Figures 8 and 9.

It can be seen from the experimental results that the reconstructed $3 \mathrm{D}$ image quality is poor with the $10 \%$ data sparsity rate, and it is difficult to identify the specific targets. However, the images can be reconstructed well even at 20\% data sparsity rate. And a larger data will result in a better image. Also, a reconstruction error $\varepsilon$ is introduced to evaluate the quality of the images reconstructed with different reconstruction algorithms:

$$
\varepsilon=\frac{\sqrt{\left(\sum_{i=1}^{n}\left|g^{\prime}(x, y, z)-g(x, y, z)\right|^{2}\right) / n^{2}}}{\sqrt{\left(\sum_{i=1}^{n}|g(x, y, z)|^{2}\right) / n^{2}}},
$$

where $g^{\prime}(x, y, z)$ is the reconstructed signal and $g(x, y, z)$ denotes the original signal. A larger $\varepsilon$ denotes a significant deviation of the reconstructed signal from the original signal and thus means a poorer performance of the reconstruction algorithm. The reconstruction error comparisons between SL0 and NSL0 under different sparsity rates are listed in Table 4.

Also, the calculation time of the algorithms is presented in Table 5. The CPU is Intel Core i5-4210 M @ 2.6 GHz, and the memory is $8 \mathrm{~GB}$.

The above results show that the presented NSL0 reconstruction algorithm has a smaller error and a fast calculation speed compared with the SL0 reconstruction algorithm. This is contributed by Newton's method in the NSL0 algorithm.

\section{Conclusions}

An algorithm for THz FMCW SAR imaging based on compressed sensing is investigated in this paper. The developed $220 \mathrm{GHz} F M C W$ imaging radar system is introduced, and the signal model is built firstly. RMA for the developed $\mathrm{THz}$ FMCW SAR is then derived. Compressed sensing is described, and the NSL0 reconstruction algorithm is presented to reconstruct signal with sparse samples. And the algorithm based on compressed sensing for the developed THz FMCW SAR is summarized. Experiments are performed to verify the presented imaging algorithms. The experimental results show that it is able to reconstruct the image well even at the sparsity rate of $20 \%$. The presented $3 \mathrm{D}$ imaging algorithm for the $\mathrm{THz}$ FMCW imaging radar system can improve the imaging efficiency by reducing the requirements for spatial data acquisition.

The developed $220 \mathrm{GHz}$ FMCW SAR imaging system has been used for nondestructive testing of composite materials in aerospace and critical structural applications. As only a single transceiver is integrated in the system, it must employ a grid-like mechanical scanning to cover an area and results in a high time cost. The imaging system can be upgraded to multiple transceivers which will have a faster imaging speed. The proposed compressed sensing image reconstruction algorithm can be also applied for sparse transceiver array configuration which is able to achieve a lower imaging system cost by reducing $\mathrm{THz}$ transceivers.

\section{Data Availability}

The data used to support this study are included within this articles as tables. If there is a need for any other information, the corresponding author may be contacted by e-mail.

\section{Conflicts of Interest}

The authors declare that there are no conflicts of interest regarding the publication of this paper.

\section{Acknowledgments}

This work was partially supported by the Science Foundation of the Jiangsu Higher Education Institutions of China (No. 18KJB413003), the High-Level Talent Foundation of Jinling Institute of Technology (Nos. Jit-b-201713, Jit-b-202029, Jitb-201816, and Jit-b-201628), the Research Fund Incubation Project of Jinling Institute of Technology (No. jit-fhxm201705), the Shanghai Science and Technology Innovation Action Plan (No. 19511108400), and the Jiangsu Province Fifth "333 High-Level Talent Training Project" Second-Level Funding Research Project (No. 2019).

\section{References}

[1] R. I. Stantchev, X. Yu, T. Blu, and E. Pickwell-MacPherson, "Real-time terahertz imaging with a single-pixel detector," Nature Communications, vol. 11, no. 1, pp. 2535-2538, 2020.

[2] D. M. Mittleman, "Twenty years of terahertz imaging [Invited]," Optics Express, vol. 26, no. 8, pp. 9417-9431, 2018. 
[3] H. Essen, A. Wahlen, R. Sommer et al., "High-bandwidth $220 \mathrm{GHz}$ experimental radar," Electronics Letters, vol. 43, no. 20, pp. 1114-1116, 2007.

[4] T. Jaeschke, C. Bredendiek, and N. Pohl, "3D FMCW SAR imaging based on a $240 \mathrm{GHz}$ SiGe transceiver chip with integrated antenna," in Proceedings of German Microwave Conference, pp. 1-4, Aachen, Germany, March 2014.

[5] K. B. Cooper, R. J. Dengler, G. Chattopadhyay et al., "A highresolution imaging radar at $580 \mathrm{GHz}$," IEEE Microwave and Wireless Components Letters, vol. 18, no. 1, pp. 64-66, 2008.

[6] K. B. Cooper, R. J. Dengler, N. Llombart et al., Fast HighResolution Terahertz Radar Imaging at 25 Meters, Terahertz Physics, Devices, and Systems IV: Advanced Applications in Industry and DefenseProceeding of SPIE, Orlando, FL, USA, 2010.

[7] K. B. Cooper, R. J. Dengler, and N. Llombart, “An approach for sub-second imaging of concealed objects using terahertz (THz) radar," Journal of Infrared, Millimeter and Terahertz Waves, vol. 30, no. 12, pp. 1297-1307, 2009.

[8] J. Jinshan Ding, M. Kahl, O. Loffeld, and P. H. Bolivar, “THz 3-D image formation using SAR techniques: simulation, processing and experimental results," IEEE Transactions on Terahertz Science and Technology, vol. 3, no. 5, pp. 606-616, 2013.

[9] A. Keil, T. Hoyer, J. Peuser, H. Quast, and T. Löffler, "Allelectronic 3D THz synthetic reconstruction imaging system," in Proceeding of 2011 36th Int. Conf. On Infrared, Millim. THz Waves (IRMMW-THz), pp. 1-2, Houston, TX, USA, October 2011.

[10] J. Moll, P. Schops, and V. Krozer, "Towards three-dimensional millimeter-wave radar with the bistatic fast-factorized backprojection algorithm-Potential and limitations," IEEE Transactions on Terahertz Science and Technology, vol. 2, no. 4, pp. 432-440, 2012.

[11] J. J. M. De Wit, A. Meta, and P. Hoogeboom, "Modified rangeDoppler processing for FM-CW synthetic aperture radar," IEEE Geoscience and Remote Sensing Letters, vol. 3, no. 1, pp. 83-87, 2006.

[12] A. Meta, P. Hoogeboom, and L. P. Ligthart, "Non-linear frequency scaling algorithm for FMCW SAR data," in Proceedings of the Eur. Radar Conf., pp. 9-12, Manchester, UK, September 2006.

[13] H. Gao, C. Li, S. Wu et al., "Study of the extended phase shift migration for three-dimensional MIMO-SAR imaging in terahertz band," IEEE Access, vol. 8, pp. 24773-24783, 2020.

[14] J. A. Tropp and A. C. Gilbert, "Signal recovery from random measurements via orthogonal matching pursuit," IEEE Transactions on Information Theory, vol. 53, no. 12, pp. 4655-4666, 2007.

[15] J. F. Determe, J. Louveaux, L. Jacques, and F. Horlin, "On the noise robustness of simultaneous orthogonal matching pursuit," IEEE Transactions on Signal Processing, vol. 65, pp. $864-875,2016$.

[16] D. L. Donoho, Y. Tsaig, I. Drori, and J.-L. Starck, "Sparse solution of underdetermined systems of linear equations by stagewise orthogonal matching pursuit," IEEE Transactions on Information Theory, vol. 58, no. 2, pp. 1094-1121, 2012.

[17] D. Needell and R. Vershynin, "Signal recovery from incomplete and inaccurate measurements via regularized orthogonal matching pursuit," IEEE Journal of Selected Topics in Signal Processing, vol. 4, no. 2, pp. 310-316, 2010.

[18] D. Needell and J. A. Tropp, "CoSaMP," Communications of the ACM, vol. 53, no. 12, pp. 93-100, 2010.
[19] H. Mohimani, M. Babaie-Zadeh, and C. Jutten, "A fast approach for overcomplete sparse decomposition based on smoothed \$lell $\{0\} \$$ norm," IEEE Transactions on Signal Processing, vol. 57, no. 1, pp. 289-301, 2009.

[20] L. Wang, X. Yin, H. Yue, and J. Xiang, "A regularized weighted smoothed Lo norm minimization method for underdetermined blind source separation," Sensors, vol. 18, no. $12,2018$.

[21] R. Zhao, W. Lin, H. Li, and S. Hu, "Reconstruction algorithm for compressive sensing based on smoothed L0 norm and revised Newton method," Journal of Computer Aided Design and Computer Graphics, vol. 24, pp. 478-484, 2012. 